\title{
Some Productive Mechanisms of Coining New Technical Terms in Developing Terminologies
}

\author{
Maxim Nikolayevich Latu ${ }^{1}$ \\ ${ }^{1}$ Pyatigorsk State Linguistic University, Western European Languages and Cultures Department, Pyatigorsk, \\ Russian Federation \\ Correspondence: Maxim Nikolayevich Latu, Pyatigorsk State Linguistic University, Western European \\ Languages and Cultures Department, Kalinin Avenue, 9, Pyatigorsk, 357532, Russian Federation. Tel: \\ 79-18-799-1816. E-mail: laatuu@yandex.ru
}

\author{
Received: October 10, 2014 Accepted: November 6, 2014 Online Published: February 25, 2015 \\ doi:10.5539/ass.v11n6p157 URL: http://dx.doi.org/10.5539/ass.v11n6p157
}

\begin{abstract}
The article considers some of the mechanisms and principles that are argued to be productive in coining technical terms in developing terminologies. The examples are mainly drawn from the fields of nanotechnology, information technologies, space research, genetics and some others. Special attention is paid to the principles of systemic organization of technical terms within a terminology and its reflection in the process of the creation of new technical terms. The structural and semantic peculiarities of one and multi-component technical terms are analyzed as well as the preferences in the number of components. The principles and types of the formation of terminological oppositions and their reflection in the underlying forms of technical terms are discussed. I also focus on the principles of choice of term elements, especially productive and systemic that builds up the corpus of "building blocks" for coining new terms for a certain terminology or the language of science on the whole. The role of productive spheres of borrowing term elements for coining new technical terms is also put into context.
\end{abstract}

Keywords: terminology, technical term, term element, coining, terminological system, semantics, structure, derivation, terminological opposition

\section{Introduction}

The interest to technical terms has a long lasting history. Some of the first dictionaries in England were the corpuses of the so-called "hard words" of different fields of professional knowledge. The Expositor was published in 1616, the famous New World of Words followed it in 1658, the pages of Dictionary of hard and technical words or terms of art contained lexemes from more than 60 fields of knowledge (Lerer, 2008). Many were of Greek and Latin origin or had borrowed elements from other languages. The fact is that some technical terms no matter how hard they are constantly enrich the vocabulary of general use even today. The principles and mechanisms of technical terms coining are in many ways specific. According to statistics more than 2000 new sciences emerged in the $\mathrm{XX}^{\text {th }}$ century and $90 \%$ of new words are representing special concepts (Grinev-Grinevich, 2008). For this reason every person knows some of the technical terms in various fields of knowledge (Crystal, 2007). The principles of coining technical terms are indeed worth attention and hard to study. Terminologies change in time, some technical terms becoming archaic, some concepts seize to be relevant, new principles of coining new technical terms may emerge (indeed far not all terminologies rely directly on Greek and Latin elements today, some stick to the patterns that they have elaborated within the field that makes them unique and distinctive in this sense (see Alimuradov \& Gorbunova, 2014; Barinova et al., 2011; Khakieva, 2013; Maznyova, 2008; Rozhdestvenskaya, 2008). The technical term methylphenidate hydrochloride will leave one in no doubt that it belongs to chemistry. However some mechanisms and principles of coining technical terms have become rather productive for developing terminologies today to which I want to pay special attention to.

\section{Methods and Tools}

Within the scope of this study I focus on some of the productive principles and mechanisms of coining technical terms. My approach is to demonstrate how they work in contemporary developing terminologies both with a long history and relatively young, from which I draw most of the examples. Among these are the terminologies 
of space research, IT, nanotechnologies, genetics, etc. being among the most promising fields of modern science (Keats, 2011; see also Shaw, 2008). On the one hand these terminologies represent fields of knowledge that differ in subject and objectives of the research, as well as characteristics including peculiarities of historical development, etc., on the other the results of the research are of high demand and they remain the priority spheres in many developed and developing countries of the world. As new concepts appear the terminology keeps expanding with more technical terms that enter the already existing hierarchy following the principles of classification described in the works of S.I. Hayakawa (S. I. Hayakawa \& A. R. Hayakawa, 1990). It has become quite obvious that the organization of concepts within the fields is inevitably reflected in the underlying forms of the coined technical terms and in some cases is related to what they express. Studying the structural organization of technical terms within the mentioned fields I also relied upon the general principles of frame analysis developed by C.J. Fillmore and other scholars (Fillmore, 1982; see also Cooper et al., 2002) as well as their application to modeling the terminology structure (Alimuradov \& Chursin, 2009; Latu, 2009; Razduev, 2013; Razduev \& Alimuradov, 2011). The technical terms were singled out from a corpus of texts including scientific papers and monographs to build data bases devoted to each of the mentioned fields of knowledge. The analysis of their definition was carried out to find the adjacent concepts. The structure of the technical terms was analyzed and the etymological analysis was also considered an important tool. The component analysis of term semantic structure helped to shed light on how the semantic structure of some technical terms forms oppositions and the necessary lexemes a chosen to be used in a structure of multi-component terms. The comparative analysis was applied to study synchronic and diachronic peculiarities of technical terms creation. Mathematical tools and the qualitative method were used throughout the study and in particular made it possible to single out productive and systemic term elements.

\section{Results}

The fact that technical terms differ by the number of constituent components is a rather obvious one. The technical terms that consist of one root are one-component and those that have more than one root in their structure are multi-component. It is remarkable to note that statistics proves that the body of multi-component terms (the majority of which are word combinations) considerably exceeds the number of one-component terms. As the analysis has shown there is a general tendency for a number of components to grow in technical terms at the current stage of terminologies development both young and old. The number of technical terms with three and more components continues to increase and the gap between the percentage of one-component and multi-component technical terms is growing larger. So one may conclude that, generally speaking, a technical term today is more a word combination rather than just a word that to some extent differentiates it from the vocabulary of general use.

\section{Discussion}

An average picture is as follows. The largest percentage is two-component technical terms. Three-component technical terms hold the second place. Their number just a little bit exceeds or is equal to that of one-component technical terms. Then as the number of components grows there is an abrupt decrease in the percentage of technical terms and the more likely there are abbreviations used as duplicates instead of the full forms of the technical terms, for example, COM-port. POHMELFS (Parallel Optimized Host Message Exchange Layered File System), etc. The comparative analysis in some studies has proved that the number of multi-component technical terms with three and more elements in relatively young terminologies such as the terminology of nanotechnology grows very fast (Razduev, 2013).

Why does it become more preferable to coin multi-component technical terms? There are several possible explanations to this fact. One is the development and complication of the academic and professional knowledge structure in various fields. Thus, the study of the history of military terminology proved the increase of abbreviations in the $\mathrm{XX}^{\text {th }}$ century. It is worth noting that three- and four-component technical terms are extremely rare among the archaic vocabulary of the terminologies that have a long history and pre-date the $\mathrm{XX}^{\text {th }}$ century. Another one is a natural demand for a technical term to reflect more dominant features of the expressed notion in the underlying form to best describe it, for example, active catalytic phase, small angle neutron scattering, plasma-enhanced chemical vapor deposition, total internal reflection fluorescence microscopy, highly active \& selective nanostructured oxide catalysts, etc. (www.nanodic.com) that are nanotechnology technical terms. Even more important in this respect is the fundamental characteristic of technical terms systemacy. It results in a demand to reflect the structural relationship with a generic or other adjacent notions in the underlying form of a technical term. Thus, one or more constituent elements of a multi-component technical term may be expressed by an already existing one- or multicomponent technical term. Thus, the technical term switching local-area network is built up of the technical terms switching and local-area network that belong 
to the same field of knowledge and are systemic term elements in its structure. It is important to mention in this respect that the tendency to coin terms with more and more complex structure is effectively counterbalanced by the demand of scholars that a technical term should have an easy-to-use form. For this reason the percentage of multi-component technical terms with two components is the largest. However the influence of this factor is weakened by the possibility to use abbreviations.

The general regularity is that generic notions are more likely to be expressed by technical terms with a smaller number of elements than superordinate concepts. The number of components grows with the volume of a notion becoming smaller and more specific. It is also worth noting that the category to which the expressed notion belongs also matters. Thus a structure with four and more components is not common and rare for technical terms that express process, characteristic or agent due to which the number of abbreviations is limited too.

\section{Terminological Oppositions}

The formation of terminological oppositions is a natural process for superordinate technical terms that structurally are on the same level of academic knowledge classification hierarchy. Superordinate notions always have a number of shared characteristics as well as some differentiae or distinguishing marks that created the necessity to distinguish them and became the basis for their contradistinction. This is directly related to the systematic character and hierarchy organization of academic knowledge.

When the underlying form of superordinate technical terms does not have an indication that points to their contradistinction the opposition is implicit, for example, pulsar and magnetar are the subtypes of neutron stars Their opposition remains invisible and unobvious in the underlying forms, as there is no sign of antonymity or gradation. It is much more interesting though, when the opposition is reflected linguistically. In this case the opposition is explicit since the opposition is clearly expressed and obvious, for example, high program address and low program address that are in explicit opposition since the classifying term elements high and low are antonymous.

Thus, the underlying form of the second technical term is in certain respects preconditioned by the underlying form of the first term, is secondary to it and together with the use of systemic term elements in multi-component technical terms is a verbal representation of the systemacy of terminology. Another peculiarity of implicit oppositions is that the second term reflects the structure of the first one as if in a mirror due to which such technical terms have the same number of term elements, for example, two-component technical terms real address and virtual address, five-component technical terms heterogeneous distributed database and homogeneous distributed database, etc.

The analysis has proved that explicit terminological oppositions can be classified into three types according to the principles of coining technical terms. In Type 1 the second term in the opposition is created by means of affixation, when a negative prefix or suffix is added to the first one, for example, mounting and unmounting. The corpus of potential affixes to be used ( $a$-, de-, un-, anti-, etc.) is universal on the whole for different terminologies. They convey information about the reverse process or the absence of a characteristic in the semantics of the second technical term in the opposition.

Type 2 opposition presumes that the technical terms are coined with the help of lexemes with antonymous meaning that become term elements, for example, local network and global network, real address and virtual address, etc. Thus, this opposition is based on the antonymous nature of the term elements.

The term elements in Type 3 opposition represent are the lexemes borrowed from a specific LSG, spectrum, category, etc. or the already existing classification in some other field of knowledge that represent the gradation of a characteristic. For example, certain colors are used as term elements astronomy: yellow dwarf, white dwarf, etc. as well as technical terms borrowed from chemistry to rely on the already existing opposition of the notions they express: helium white dwarf, neon-magnesium white dwarf, etc. It is clear that the coining of technical terms to form oppositions of Type 2 and 3 relies upon the existing logical relations that have already been expressed in the language. The number of potential superordinate technical terms in the opposition may vary.

Some term elements that are used to coin technical terms to form explicit opposition may become productive in this function within a terminology or some terminologies, for example, the term elements static and dynamic are productive to form oppositions in IT terminology: static typification and dynamic typification, static random access memory and dynamic random access memory, static web-page and dynamic web-page, etc.

\section{Productive and systemic technical term elements}

A technical term is a constituent part of a terminology while a term element is a smallest meaningful component in a technical term structure and represents a building block used to coin the term. Thus, it is a lexeme or its part 
in a technical term structure that is its integral component. Its semantics become the underlying form in motivated technical terms due to what the choice of a proper lexeme to coin a new term is essentially important.

A specific body of productive technical term elements may be sorted out of the corpus of all the technical term elements that belong to a certain terminology. A productive term element, in this respect, is a word or a word combination that is used as a term element in the structure of two or more technical terms, for example, the lexeme text was used to coin the following IT technical terms: text format, text editor, text processor, text file, text user interface, etc., being a productive building block for creating IT terms. The body of such term elements may shed light on the certain semantics that become preferable for scholars of a particular field to appeal to when a necessity comes to coin a new term to describe a new notion. A very obvious and expected as well as the most numerous productive term element to coin technical terms in the field of nanotechnology is, of course, nano-. The term element transgenic is productive for and characteristic of technical terms in genetics, for example, transgenic organism, transgenic hybridoma, transgenic line, transgenic marker, etc.

The corpus of productive term elements also includes a very specific body of the so called systemic term elements. A systemic term element is a term element that functions in a terminology as an independent technical term and has become the basis for the creation of new multi-component technical terms. All the term elements that are not individual technical terms within this terminological system are non-systemic. Thus, for example, the structure of the technical term text interface (as well as graphical user interface, semantic interface, command interface, medium dependent interface, SILK-interface, etc.) contains and is based on the systemic term element interface that function as an independent technical term in IT terminology while the lexeme text and its derivatives are not individual technical terms within this terminology and thus is non-systemic. The technical term nanotube in Nanotechnology also function as a systemic term element in the structure of many terms that represent its variations, such as carbon nanotube, multi-wall nanotube, etc. Thus, the systemic term elements are frequent in the structure of hyponyms that express superordinate notions.

It is worth noting that the functioning of productive elements is generally restricted to the technical terms only. Thus, they are not used in coining professional jargon words (colloquial duplicates of technical terms that express the same notions but are not used in the official academic language) or professional slang words.

The demand to reflect the already existing pieces of knowledge that belong to the particular field and in the underlying form of a technical term and rely on the bank of language units that have already been used leads to the fact that some multi-component technical terms are totally built up on the productive term elements both systemic and non-systemic, for example, personal communications service, IP-address, switching local area network, etc. the term elements of which are productive for IT.

The comparative analysis of such corpuses of productive term elements of different terminologies allows to single out the body of the most productive term elements that are used to coin technical terms in various terminologies and, thus, have specific semantics that makes them preferential universal building blocks for academic language in general, among these are the term elements one, multi-, system, etc.

\section{Productive Spheres of Term Elements Borrowing}

Productive and systemic term elements are important when it comes to coining new technical terms, but there is another important aspect related to term elements which is productive spheres of borrowing term elements. The latter may be an LSG, semantic fields or other terminologies the lexemes of which are frequently used to coin new terms in a particular terminology. These spheres become distinctive language-donors that are appear preferential and open to supply the lexemes to become the term elements in the structure of technical terms. For example, IT borrows term elements from a sphere related to language study and communication: language processor, language module, text interface, hypertext, syntax, syntax error, command line, operational semantics, content management system, script, page, speech technology, etc. Sometimes a sphere of borrowing may be productive not for the terminology on the whole but some parts of it, for example, the names of predators or just strong animals and birds of prey are readily used respectively in artillery and military aviation, for example, tiger IV, fighting falcon, etc. Animal names as well as mythical heroes and creatures are all around in astronomy: blue giant, cancer (constellation), crab nebula, T Tauri, cepheids, etc.

\section{Conclusion}

The analysis of the corpuses of the technical terms helped to define some principles and mechanisms that to some extent influence and direct the process of coining new terms in developing terminologies. Thus, technical terms tend to be multi-component rather than one-component with a growing number of components trend effectively counterbalanced by an easy-to-use technical term demand. The oppositional nature of superordinate 
concepts also tends to be reflected in the underlying forms of the technical terms by means of the defined mechanisms. So do the principles of hierarchical classification of pieces of knowledge in a field that results in the use of systemic term elements representing the relations with hyperonym or adjacent concepts. Each field develops a corpus of productive term elements that serve as convenient building blocks for creating new language units expressing the specifics of knowledge. Having certain semantic fields as donors of term elements may also become characteristic. A field of personal names keep supplying many terminologies due to what the number of eponymic technical terms also increases (for example in nanotechnology) and so do place names in the structure of terms for different species in zoology. It is pretty clear that all terms do not and cannot naturally follow only a few patterns and there will always be exceptions. Still the abovementioned principles appear as regularities in coining technical terms in developing terminologies. The prospects of the research are the further analysis of the productive mechanisms of coining new technical terms in different fields of human knowledge, including both new and old ones.

\section{Acknowledgements}

The authors express their gratitude to Ministry of education and science of the Russian Federation that financially supported the study (projects 14.Z56.14.4389-MK; 2014-2016 state assignment to the universities)

\section{References}

Alimuradov, O. A., \& Chursin, O. V. (2009). Cognitive-frame approach to the functional-semantic characteristics of the modern English music lexis. In the world of scientific discoveries, 3-1, 21-28.

Alimuradov, O. A., \& Gorbunova, N. N. (2014). Some basic syntactic word-formation models, realized in the English management terminology. Proceedings of higher education institutions. Humanities series, 5(2), 95-101.

Alimuradov, O. A., Blinova, D. E., \& Razduev, A. V. (2010). Frame modeling of the linguistic picture of the world, represented in the English child's discourse. Irkutsk State Linguistic Bulletin, 4, 6-13.

Barinova, Y. Y., Svionkovskaya, S. V., \& Konovalova, A. V. (2011). Terms and professional metaphors in the stock-exchange informational-analytical discourse: German, Spanish, French and Russian language. Pyatigorsk State Linguistic University Bulletin, 4, 191-197.

Cooper, R. P., Yule, P., Fox, J., \& Glasspool, D. W. (2002). Modeling High-Level Cognitive Processes. Mahwah: Lawrence Erlbaum Associates (p. 428).

Crystal, D. (2007). Words, Words, Words (p. 224). Oxford: Oxford University Press.

Fillmore, C. J. (1982). Frame semantics. In Lim (Ed.), Linguistics in the morning calm: Selected papers from the SICOL-1981 (pp. 111-137). Seoul: Hanship.

Gekkiev, K. B. (2010). The analysis of productivity of some means of term formation drawing on the material of the English criminological lexis. Pyatigorsk State Linguistic University Bulletin, 1, 81-83.

Grinev-Grinevich, S. V. (2008). Terminology study (p. 304). Moscow: Academy.

Hayakawa, S. I., \& Hayakawa, A. R. (1990). Language in thought and action (p. 196). San Diego: Harcourt.

Keats, J. (2011). Virtual Words: Language on the Edge of Science and Technology (p. 190). Oxford: Oxford University Press.

Khakieva, Z. U. (2013). The English terminology of construction and construction technologies: Structure, semantics and dynamics: Ph.D. thesis in Philology. Pyatigorsk (p. 225).

Latu, M. N. (2009). The English military terminology in its historical development: Structural-semantic and cognitive-frame aspects: Ph.D. thesis in Philology. Rostov-on-Don (p. 191).

Lerer, S. (2008). The history of the English language (2nd ed., Part 1, 2, 3, p. 196). Chantilly: The Teaching Company.

Maznyova, Y. A. (2009). The cognitive-semantic characterization of juridical abbreviated terms in the modern English language. Pyatigorsk State Linguistic University Bulletin, 1, 177-181.

Nanodic.com. All About Nano. Retrieved September 12, 2014, from http://www.nanodic.com

Razduev, A. V. (2013). Contemporary English nanotechnology terminology: Structural, semantic, cognitive-frame and lexicographic models: Ph.D. thesis in Philology. Pyatigorsk (p. 242).

Razduev, A. V., \& Alimuradov, O. A. (2011). The experience of cognitive modeling and lexicographying of the 
English nanotechnology sublanguage. Proceedings of the Southern Federal University. Philology, 1, 72-86.

Rozhdestvenskaya, S. V. (2008). The influence of the foreign lexis on the replenishment of the terminological funds of the English and Russian languages (drawing on the example of the ceramics terminology). Pyatigorsk State Linguistic University Bulletin, 4, 78-82.

Shaw, D. B. (2008). Techno culture: The Key Concepts (p. 218). New York: Berg.

\section{Copyrights}

Copyright for this article is retained by the author(s), with first publication rights granted to the journal.

This is an open-access article distributed under the terms and conditions of the Creative Commons Attribution license (http://creativecommons.org/licenses/by/3.0/). 